\title{
Clúster de síntomas en insuficiencia cardiaca avanzada: una revisión sistemática
}

Symptom Cluster among Patients with Advanced Heart Failure: A Systematic Review

Grupo de sintomas em insuficiência cardíaca avançada: uma revisão sistemática

Como citar este artículo

Pérez Sandoval Leidy Paola, Moreno García Jenny Rocío, Barboza Galindo Adriana Paola. Clúster de síntomas en insuficiencia cardiaca avanzada: una revisión sistemática. Revista Cuidarte. 2021;12(2):e1302.

http://dx.doi.org/10.15649/cuidarte.1302

Revista Cuidarte

Rev Cuid. May - Ago 2021; 12(2): e1302

doij http://dx.doi.org/10.15649/cuidarte.1302

E-ISSN: 2346-3414

(1) Leidy Paola Pérez Sandoval ${ }^{1}$

(1) Jenny Rocío Moreno García²

(1) Adriana Paola Barboza Galindo 3

1 Universidad El Bosque, Bogotá D.C, Colombia. Autor de Correspondencia. E-mail: Iperezsa@unbosque.edu.co

2 Universidad El Bosque, Bogotá D.C, Colombia.

E-mail: jrmoreno@unbosque.edu.co

3 Universidad El Bosque, Bogotá D.C, Colombia.

E-mail: abarboza@unbosque.edu.co

\section{Resumen}

Introducción: La Insuficiencia cardiaca avanzada genera deterioro clínico y funcional marcado en el paciente. A través del tiempo se han desarrollado investigaciones para evaluar síntomas y aliviar el sufrimiento. El presente artículo tiene como objetivo identificar los clúster de síntomas en pacientes con insuficiencia cardiaca avanzada, que puedan contribuir en la consolidación del conocimiento. Materiales y Métodos: Una estrategia PICO estableció la pregunta clave de revisión desarrollada, mediante PRISMA, con búsquedas en: Scopus, Pubmed, Embase y Google Scholar. Se incluyeron artículos de fuente primaria, insuficiencia cardiaca avanzada, publicados 2010 - 2019 en inglés y español, se excluye población pediátrica. Se realizaron análisis descriptivos de los síntomas encontrados. Resultados: De 8000 estudios, fueron incluidos 11 para revisión completa, la mayoría de corte transversal, cuyas características de la muestra corresponde $50 \%$ hombres, entre 56 y 83 años. Se identificaron en el clúster físico síntomas: fatiga, dificultad para dormir, disnea; en el cluster emocional se identifican: Depresión, deterioro cognitivo y preocupación, además se encontró evaluación de síntomas por región geográfica. Discusión: Existe un consenso en la clasificación de síntomas en grupos denominados físico y emocional, llama la atención que el edema en algunos casos no está incluido en ellos. Limitaciones: Se incluyeron dos estudios del mismo autor en diferente año de publicación, población y los clúster son los mismos, pero con análisis de variables como calidad de vida y estado funcional. Conclusión: Diversidad de poblaciones, tipos de estudio y métodos de análisis, no permiten un único enfoque para agrupación de los síntomas más frecuentes en pacientes con insuficiencia cardiaca avanzada.

Palabras clave: Evaluación de Síntomas, Insuficiencia Cardiaca, Síndrome.

Recibido: junio 12 de 2020

Aceptado: diciembre 17 de 2020

Publicado: mayo 18 de 2021 $\square *$ Correspondencia Leidy Paola Pérez Sandoval E-mail:Iperezsa@unbosque.edu.co 


\title{
Symptom Cluster among Patients with Advanced Heart Failure: A Systematic Review
}

\begin{abstract}
Introduction: Advanced heart failure leads to severe clinical and functional deterioration in patients. Over the years, research has been focused on the evaluation of symptoms and alleviation of suffering. This study aims to identify symptom clusters among patients with advanced heart failure in order to contribute towards knowledge consolidation. Materials and Methods: The PICO strategy helps define the key review question developed through PRISMA, involving searches in Scopus, PubMed, Embase and Google Scholar. Primary source articles on advanced heart failure which had been published between 2010 and 2019 in English and Spanish, excluding the pediatric population, were considered for this review. We carried out a descriptive analysis of the symptoms found in the articles. Results: A total of 11 out of 8000 studies were selected for a complete review, most of them are cross-sectional studies in which sample characterization corresponds to $50 \%$ of men aged 56 to 83 years. Fatigue, sleeping difficulty and dyspnea were some of the symptoms found in the physical symptom cluster, while depression, cognitive deterioration and concern were found in the emotional symptom cluster. Evaluations of symptoms by geographic region were also found. Discussion: There is a general consensus about the classification of symptoms in physical and emotional clusters. However, it is surprising to find out that edema is not included in any of them. Limitations: Two studies from the same author but published in different years were included in the review as the population and clusters were the same but different variables such as quality of life and functional status were studied. Conclusion: Having diverse populations, study types and analysis methods do not allow applying a single approach to grouping the most frequent symptoms in patients with advanced heart failure.
\end{abstract}

Key words: Symptom Assessment, Heart Failure, Syndrome.

\section{Grupo de sintomas em insuficiência cardíaca avançada: uma revisão sistemática}

\section{Resumo}

Introdução: A insuficiência cardíaca avançada gera deterioração clínica e funcional marcado no paciente. Foi desenvolvida ao longo do tempo pesquisas para avaliar os sintomas e aliviar o sofrimento. $\mathrm{O}$ presente artigo tem como objetivo identificar os agrupamentos de sintomas em pacientes com insuficiência cardíaca avançada, que podem contribuir para a consolidação do conhecimento. Materiais e Métodos: Uma estratégia PICO estabeleceu a questão-chave de revisão desenvolvida, usando PRISMA, com buscas em: Scopus, Pubmed, Embase e Google Scholar. Artigos de uma fonte primária foram incluídos, insuficiência cardíaca avançada, publicados em 2010 - 2019 em inglês e espanhol, a população pediátrica é excluída. Foram realizadas análises descritivas dos sintomas encontrados. Resultados: Dos 8.000 estudos, 11 foram incluídos para revisão completa, a maioria de corte transversal, cujas características da amostra correspondem a 50\% de homens, entre 56 e 83 anos. Foram identificados no agrupamento físico os sintomas: cansaço, dificuldade para dormir, dispneia; no agrupamento emocional, foram identificados: Depressão, deterioração cognitiva e preocupação, assim como foi encontrada uma avaliação dos sintomas por região geográfica. Discussão: Existe um consenso na classificação dos sintomas em grupos denominados físico e emocional, chama a atenção que o edema em alguns casos não está incluído neles. Limitações: Foram incluídos dois estudos do mesmo autor em diferentes anos de publicação, a população e os agrupamentos são os mesmos, mas com análise de variáveis como qualidade de vida e estado funcional. Conclusão: A diversidade de populações, tipos de estudos e métodos de análise não permitem uma abordagem única para agrupar os sintomas mais frequentes em pacientes com insuficiência cardíaca avançada.

Palavras chave: Avaliação de Sintomas, Insuficiência Cardíaca, Síndrome. 


\section{Introducción}

La insuficiencia cardiaca (IC) es un problema de salud pública ${ }^{1}$, se caracteriza por un compromiso circulatorio clínicamente significativo; que produce un desequilibrio entre la capacidad del corazón de bombear sangre y las necesidades del organismo 2. Según la American College of Cardiology, las proyecciones de la enfermedad tendrán un aumento de un $46 \%$ para el $2030^{3}$, afectando a 26 millones de personas, mayores de 70 años a nivel mundial ${ }^{4}$.

La insuficiencia cardiaca avanzada es la principal causa de hospitalizaciones recurrentes debido a un deterioro clínico, deterioro funcional marcado y los síntomas refractarios que el paciente presenta, se encuentran entre los predictores más destacados, que nos indican que el paciente se acerca al fin de vida ${ }^{5}$. Por otra parte, la American Heart Association (AHA) y el American College of Cardiology (ACC), proponen cuatro estadios en la clasificación New York Heart Association (NYHA), son utilizadas en la práctica clínica, siendo el estadio III y IV los que definen la enfermedad avanzada, asociado a un mal pronóstico en el paciente; el estadio III se define como: el paciente presenta una gran limitación en la actividad física, no presenta síntomas en reposo, pero cualquier actividad física puede provocarlos y el estadio IV: el paciente presenta incapacidad para realizar actividad física, síntomas incluso en reposo ${ }^{6,7}$. Con referencia a lo anterior se evidencia la necesidad de integrar los cuidados paliativos, esto permite realizar intervenciones holísticas, enfocadas a disminuir el sufrimiento en el paciente y familia, con el fin mejorar la calidad de vida ${ }^{8}$.

La insuficiencia cardiaca actualmente no tiene cura y puede llegar a empeorar, a pesar de estar recibiendo tratamiento y terapias óptimas, por lo cual, el paciente puede llegar a percibir una variedad de síntomas como: disnea, fatiga, dolor y depresión . Los pacientes con insuficiencia cardiaca se caracterizan por experimentar síntomas múltiples que se encuentran relacionados entre sí, estos presentan un efecto sinérgico que es considerado como predictor de mortalidad $^{10}$, por tal motivo, es necesario identificar los grupos de síntomas que se definen como : dos o más síntomas relacionados entre sí y que se presentan juntos ${ }^{11}$, adicionalmente se considera que abordar los grupos de síntomas traería mayores beneficios en comparación con el manejo de síntomas individuales ${ }^{12}$, por consiguiente, al estar relacionados entre sí y al tratar uno de los síntomas, se logra aliviar los demás que se encuentren presentes ${ }^{13}$.

Resulta importante realizar una evaluación objetiva de los síntomas del paciente, para lo cual, sobresale la teoría de los síntomas desagradables de Elizabeth Lenz, en que los síntomas desagradable deben evaluarse en todas las dimensiones: en el tiempo (duración y frecuencia), la calidad (naturaleza del síntoma), la intensidad (grado, fuerza o severidad) y el nivel de angustia (grado en el cual la experiencia del síntoma es molesta para la persona) 14,15, así mismo indica que en la aparición de grupos de síntomas es probable que resulte una experiencia multiplicativa en lugar de aditiva ${ }^{14}$. 
La literatura evidencia limitada aplicación de aspectos conceptuales y teóricos en la práctica, que puede llegar a ser superado, si inicialmente desde el ámbito clínico se realiza la agrupación de síntomas concurrentes en los pacientes de insuficiencia cardiaca avanzada, permitiendo optimizar los recursos terapéuticos. Por lo anterior, esta revisión sistemática tiene como objetivo identificar los clúster de síntomas en el paciente con insuficiencia cardiaca avanzada, para así contribuir en la consolidación del conocimiento.

\section{Materiales y Métodos}

Se realizó una revisión sistemática teniendo en cuenta los lineamientos de informes para revisiones sistemáticas y metaanálisis PRISMA ${ }^{17}$, que propone los siguientes pasos: identificación, cribado, idoneidad e inclusión, la investigación de los artículos fue realizada en el primer semestre del 2020, sobre cluster de síntomas en insuficiencia cardiaca avanzada, utilizando los motores de búsqueda de Embase, Scopus, Pubmed y Google académico, se realizó inicialmente una búsqueda manual de articulos, para posteriormente hacer la selección conjunta de aquellos artículos que fueron obtenidos en la búsqueda sistemática.

Se estableció como pregunta clave de investigación: ¿Cuáles son los clúster de síntomas que presentan los pacientes con Insuficiencia cardiaca avanzada? desarrollada bajo la estrategia PICO: P: pacientes con insuficiencia cardiaca avanzada, l: evaluación de síntomas, C: sin comparación y O: control de síntomas. La búsqueda realizada en la base de datos Medline, utilizó el siguiente logaritmo de búsqueda : ("HEART FAILURE"[All Fields] AND“SYMPTOM ASSESSMENT"[All Fields]) OR "SYMPTOM CLUSTERS"[All Fields].

\section{Criterios de selección}

Los artículos incluidos en la revisión, fueron elegibles cumpliendo los siguientes criterios de inclusión: Pacientes con diagnóstico de insuficiencia cardiaca avanzada,artículos de texto completos que estuvieran disponibles en línea, artículos en inglés o en español, artículos publicados a partir del 2010 al 2019, búsquedas limitadas a humanos, artículos fuente primaria, como parte de los criterios de búsqueda no se hace restricción del tiempo para hacer la búsqueda, Se excluyeron de la selección: Revisiones sistemáticas, estudios con población pediátrica.

\section{Selección de Artículos}

En la selección se identificó un total de 8000 documentos, los artículos fueron revisados de forma independiente por los investigadores, teniendo en cuenta los criterios de selección ya indicados anteriormente, así mismo se tuvo en cuenta para el inicio de la búsqueda el título y el resumen de los estudios para su selección, que fueron un total de 17 artículos, luego se realizó la comparación de los resultados obtenidos, hasta alcanzar una lista final unificada de 11 artículos en texto completo, los desacuerdos, fueron definidos en consenso. En la Figura 1 se muestra un diagrama de flujo de búsqueda de literatura conforme a las pautas de PRISMA, estos estudios de la revisión sistemática mostraron un total de 7045 pacientes con diagnóstico de insuficiencia cardiaca, donde hubo más predominio en hombres con más de un $50 \%$ del total de la población.

\section{Extracción de Síntesis de datos}

Para la extracción y síntesis de datos, se realizó una base de datos con los artículos de texto completo que fueron seleccionados conjuntamente por los investigadores, y que 
presenta las siguientes variables: título del artículo, autor, año de publicación, objetivo, tipo de estudio, metodología que incluye: población, muestra, criterios de inclusión, criterios de exclusión, instrumentos de medición, análisis estadístico; descripción sociodemográfica que incluye: personas analizadas, género, raza, edad, estado civil, ocupación, descripción clínica y comorbilidades, síntomas, clúster de síntomas, resultados y conclusiones, se realizó la revisión conjunta, el desacuerdo entre los investigadores se resolvió mediante la discusión conjunta.

\section{Riesgo de sesgo en los estudios individuales}

Durante la revisión de los estudios se encontraron las siguientes limitaciones: los hallazgos pueden no ser generalizables porque la selección de la muestra fue por conveniencia ${ }^{12,18,22}$, así mismo una variable importante está relacionada con el tamaño de la muestra, existe poca orientación en la literatura sobre el tamaño de la muestra en los análisis de conglomerados ${ }^{25}$. De la misma manera, en estudios transversales, no se pudo establecer la causalidad, ni examinar la estabilidad de los grupos de síntomas a lo largo del tiempo ${ }^{11,12,20}$.

\section{Análisis}

Para la revisión sistemática se tuvo en cuenta 15 clúster de síntomas, que fueron analizados por su frecuencia, de acuerdo a lo reportado por los estudios incluidos en la revisión, realizando una breve descripción y su impacto en el manejo de los cuidados paliativos, con el fin de aliviar los síntomas y mejorar la calidad de vida del paciente y su familia.

Para evitar el riesgo de sesgo entre los estudios que inducen a la comunicación selectiva, se verificó que ninguna de las autoras tenga un interés comercial con el desarrollo de esta revisión sistemática.

\section{Calidad de los artículos seleccionados}

La estrategia de búsqueda de información y la evaluación de la calidad metodológica de los estudios revisados, evidencian la calidad de los estudios incluidos, en consecuencia, esta se realizó mediante la utilización de informes de observación de criterios de estudios en epidemiología analítica observacional $\mathrm{STROBE}^{26}$, la lista de verificación está compuesta por 22 ítems. Se dio un puntaje de un 1 punto a cada criterio de verificación de la lista, teniendo un total de 22 puntos, esta revisión tuvo una variación de un puntaje bajo de 16 puntos a un puntaje alto de 19 puntos, teniendo que la media del puntaje de calidad fue de 17 puntos.

\section{Consideraciones éticas}

El presente estudio corresponde a una revisión sistemática de la literatura, no se somete a la aprobación del Comité de Ética de la Universidad El Bosque, sin embargo se tuvieron en cuenta las normas de citación correspondientes a derechos de autor, lo que hace que los autores garanticen la originalidad del texto completo.

\section{Resultados}

Esta revisión sistemática identificó un total de 8000 documentos, de los cuales 3040 corresponden a la búsqueda de bases de datos y 4960 corresponden a datos de otras búsquedas, así mismo, se eliminaron 127 registros duplicados, 7856 fueron excluidos basados en sus títulos o resúmenes, 
17 fueron sometidos a revisión de texto completo, seleccionando 11 para la revisión final, el diagrama de flujo del procedimiento de selección y su resultado, según PRISMA, se muestra en la Fig. 1

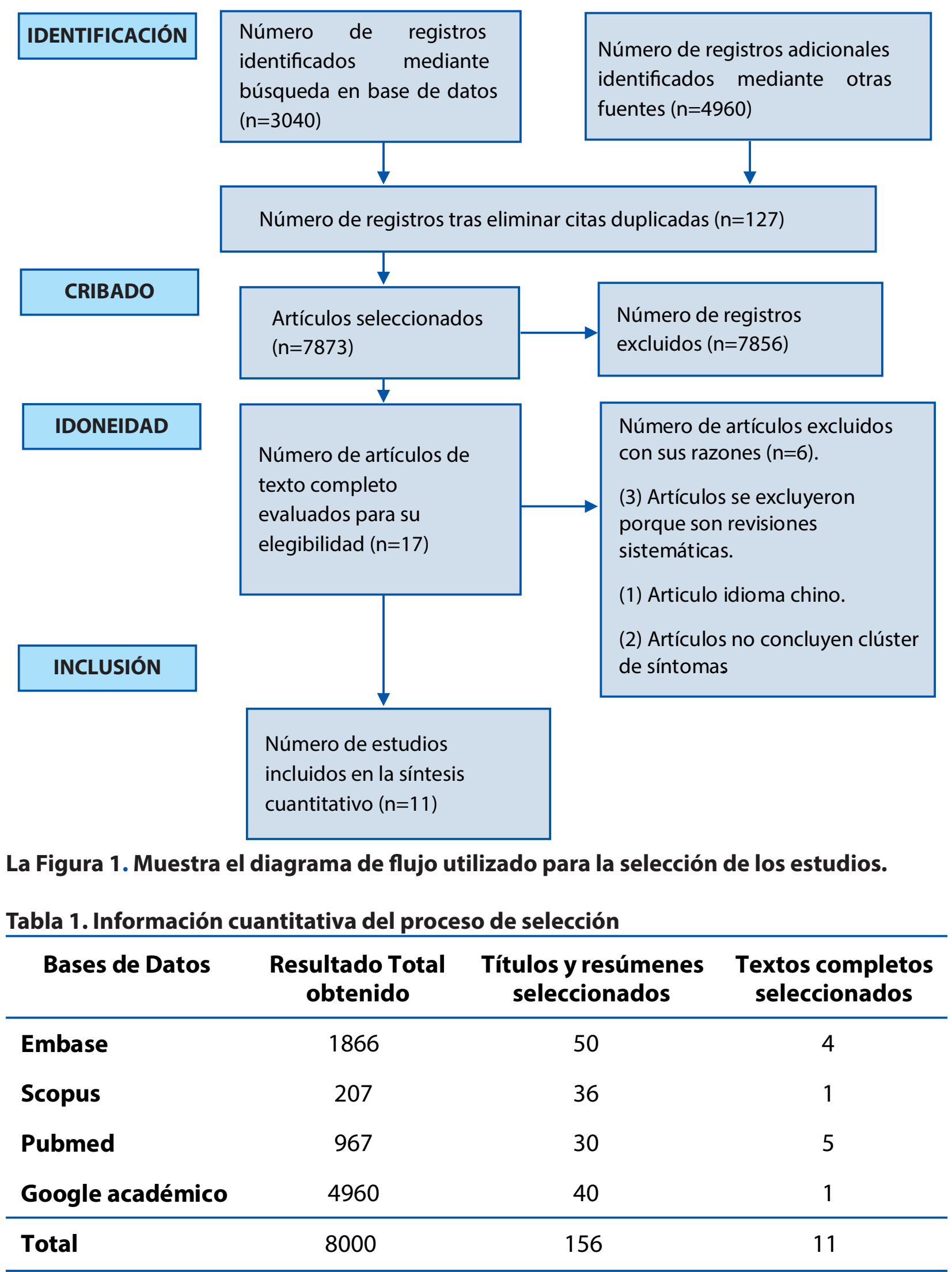


La Tabla 1, presenta en forma cuantitativa, los resultados obtenidos mediante la búsqueda en las cuatro [4] bases de datos, que se realizó mediante la selección de títulos completos.

\section{Diseño de estudio}

En lo referente al diseño de los estudios reportados en los artículos, se encontró que el 63\% fueron de corte transversal, uno de ellos resultó ser una prueba piloto, así mismo, el 27 \% de los artículos fueron de estudios longitudinales prospectivos, y sólo el $9 \%$ fueron casos y controles.

\section{Tabla 2. Recopilación de información y resultados de los estudios de clústers de síntomas} en insuficiencia cardiaca avanzada.

\begin{tabular}{|c|c|c|c|c|c|}
\hline $\begin{array}{l}\text { Autor, } \\
\text { año, } \\
\text { país }\end{array}$ & $\begin{array}{l}\text { Muestra: } n ; \\
\text { edad; } \\
\% \text { hombres (H) }\end{array}$ & Objetivo & $\begin{array}{c}\text { Métodos } \\
\text { Estadísticos }\end{array}$ & Clusters de Síntomas & Hallazgos clave \\
\hline $\begin{array}{l}\text { Salyer J, } \\
\text { et al, } \\
2019, \\
\text { Estados } \\
\text { Unidos }\end{array}$ & $\begin{array}{l}\mathrm{n}=146 \\
56 \text { años } \\
62 \% \mathrm{H}\end{array}$ & $\begin{array}{l}\text { Identificar } \\
\text { síntomas que } \\
\text { forman grupos y } \\
\text { evaluar el } \\
\text { impacto de los } \\
\text { grupos de } \\
\text { síntomas de la IC } \\
\text { en la (QOL). }\end{array}$ & $\begin{array}{l}\text { Correlación Pearson's r, } \\
\text { Análisis de componentes } \\
\text { principales (PCA), } \\
\text { Análisis de regresión }\end{array}$ & $\begin{array}{l}\text { Tres clústeres ( } 10 \text { síntomas) } \\
\text { - Comportamiento de la enfermedad } \\
\text { (ansiedad, síntomas de depresión, } \\
\text { somnolencias diurnas, Función } \\
\text { cognitiva, fatiga) } \\
\text { - Molestias gastrointestinales (apetito y } \\
\text { hambre) } \\
\text { - Malestar de la enfermedad (disnea, } \\
\text { edema y dolor) }\end{array}$ & $\begin{array}{l}\text { - La QOL influencia negativa al grupo } \\
\text { de síntomas de } \\
\text { comportamiento. } F=75,12, p<0,0001 \text {, } \\
\text { R2 }=0,404\end{array}$ \\
\hline
\end{tabular}

\begin{tabular}{|c|c|c|c|c|c|}
\hline $\begin{array}{l}\text { Quin E, } \\
\text { et al, } \\
2019 \text {, } \\
\text { Estados } \\
\text { Unidos }\end{array}$ & $\begin{array}{l}\mathrm{n}=274 \\
57.2 \pm 13.2 \text { años } \\
61 \% \mathrm{H}\end{array}$ & $\begin{array}{l}\text { Identificar } \\
\text { asociaciones } \\
\text { entre los grupos } \\
\text { de dos } \\
\text { estudios de } \\
\text { cohortes adultos } \\
\text { con IC, lgrupos } \\
\text { de síntomas } \\
\text { físicos y } \\
\text { afectivos se } \\
\text { compararon } \\
\text { mediante } \\
\text { modelos de } \\
\text { clasificación } \\
\text { cruzada. }\end{array}$ & $\begin{array}{l}\text { Modelado de mezclas de } \\
\text { clases, estadística } \\
\text { comparativa ( Kendall's, } \\
\text { tau-b y chi2 ), T-student's }\end{array}$ & $\begin{array}{l}\text { Dos clúster (4 síntomas) } \\
\text { - Síntomas físicos(disnea, trastorno del } \\
\text { sueño-vigilia,) } \\
\text { - Síntomas afectivos (depresión, } \\
\text { ansiedad) } \\
\\
\text { Subgrupos: } \\
\text { Congruente-leve }(69,3 \%) \\
\text { Incongruente }(13,9 \%) \\
\text { Congruente-grave }(16,8) \text {. }\end{array}$ & $\begin{array}{l}\text {-Subgrupo de incongruentes fue el de } \\
\text { peor supervivencia libre de } \\
\text { eventos cardiovasculares a } 180 \text { días, } \\
\text { valor de } \mathrm{P} 0,014 \text {. } \\
\text { - Los determinantes estadísticamente } \\
\text { significativos entre el subgrupo } \\
\text { entre incongruentes y congruentes } \\
\text { severos, se encuentran la } \\
\text { prescripción de antagonistas de } \\
\text { aldosterona y antidepresivos / } \\
\text { ansiolíticos. } \\
\text { - Las interacciones entre los grupos } \\
\text { físicos y afectivos se deben tener } \\
\text { en cuenta para identificar pacientes } \\
\text { con mayor riesgo de resultados } \\
\text { negativos. }\end{array}$ \\
\hline $\begin{array}{l}\text { Huang } \\
\text { TY, } \\
\text { et al, } \\
\text { 2017, } \\
\text { Taiwán }\end{array}$ & $\begin{array}{l}\mathrm{n}=258 \\
61,2 \text { años } \\
75 \% \mathrm{H}\end{array}$ & $\begin{array}{l}\text { Definir el grupo } \\
\text { de síntomas y la } \\
\text { asociación con } \\
\text { la supervivencia } \\
\text { libre de } \\
\text { hospitalizacio- } \\
\text { nes y } \\
\text { mortalidad por } \\
\text { todas las } \\
\text { causas de IC. }\end{array}$ & $\begin{array}{l}\text { Análisis de } \\
\text { conglomerados, } \\
\text { regresión } \\
\text { logística multinomial } \\
\text { Kaplan-Meier y Long } \\
\text { Rank. }\end{array}$ & $\begin{array}{l}\text { Tres clúster (6 síntomas) } \\
\text { - Sin síntomas (puntuaciones=0.7-1.5) } \\
\text { - Síntomas atípicos ( disnea y fatiga } \\
\text { puntuaciones =1.6-1.1) } \\
\text { - Síntomas típico (puntuacio- } \\
\text { nes=1.7-3-7) } \\
\text { Basados en la intensidad/puntuación } \\
\text { de: disnea, } \\
\text { fatiga, edema, insomnio, anorexia y } \\
\text { mala memoria }\end{array}$ & $\begin{array}{l}\text { - Mayor tasa de eventos a } 1 \text { año en el } \\
\text { grupo de síntomas típicos y } \\
\text { atípicos comparado con grupo sin } \\
\text { síntomas. } \\
\text { - NYHA clase III/ IV mayor probabilidad } \\
\text { presentar síntomas típicos } \\
(\mathrm{OR}=6.53 ; \mathrm{p}<0.01) \text { o síntoma atípico } \\
(\mathrm{OR}=7.01 ; \mathrm{p}<0.001) \text { grupo } \\
\text { que sus pares NYHA clase } \mathrm{I} / \mathrm{Il} \text {. } \\
\text { - Los síntomas físicos y psicosociales, } \\
\text { no se discriminaron en cada } \\
\text { clúster de síntomas. }\end{array}$ \\
\hline $\begin{array}{l}\text { Park J, } \\
\text { et al, } \\
2017, \\
\text { Estados } \\
\text { Unidos }\end{array}$ & $\begin{array}{l}\mathrm{n}=4011 \\
64,47 \text { años } \\
57.2 \% \mathrm{H}\end{array}$ & $\begin{array}{l}\text { - Identificar } \\
\text { distintas clases } \\
\text { latentes de } \\
\text { síntomas de IC. } \\
\text { - Explorar } \\
\text { influencia de las } \\
\text { características } \\
\text { sociodemográfi- } \\
\text { cas y grupo de } \\
\text { síntomas. }\end{array}$ & $\begin{array}{l}\text { Análisis de } \\
\text { conglomerados, análisis } \\
\text { de clase latente (BLRT) y } \\
\text { entropía, regresión } \\
\text { logística multinomial, } \\
\text { modelo ajustado }\end{array}$ & $\begin{array}{l}\text { Dos clúster (8 síntomas) } \\
\text { - Síntomas psicológicos (depresión, } \\
\text { preocuparse, problemas cognitivos). } \\
\text { - Síntomas físicos (edema, fatiga con } \\
\text { necesidad de reposo, fatiga con baja } \\
\text { energía, falta de aliento y dificultad } \\
\text { para dormir.) }\end{array}$ & $\begin{array}{l}\text { - Síntomas físicos y psicológicos no } \\
\text { son independientes en la IC. } \\
\text { - Valor de } p \text {, significativo en todos los } \\
\text { síntomas. }\end{array}$ \\
\hline $\begin{array}{l}\text { Doris SF } \\
\text { Yu, } \\
\text { et al, } \\
2016, \\
\text { Hong } \\
\text { Kong }\end{array}$ & $\begin{array}{l}\mathrm{n}=119 \\
82,9 \text { años } \\
59 \% \mathrm{H}\end{array}$ & $\begin{array}{l}\text { Identificar los } \\
\text { grupos de } \\
\text { síntomas en IC } \\
\text { avanzada y } \\
\text { su relación con } \\
\text { la QOL de } \\
\text { los pacientes }\end{array}$ & $\begin{array}{l}\text { Correlación de Pearson, } \\
\text { análisis de componentes } \\
\text { principales, Índice de } \\
\text { Kaiser-Myer-Olkin } \\
\text { (KMO) y análisis de } \\
\text { regresión. }\end{array}$ & $\begin{array}{l}\text { Tres clúster ( } 9 \text { síntomas) } \\
\text { - Síntomas angustiosos (disnea, } \\
\text { depresión y ansiedad). } \\
\text { - Síntomas de descondicionamiento } \\
\text { (fatiga, náuseas, } \\
\text { somnolencias diurnas, apetito } \\
\text { reducido). } \\
\text {-Síntomas molestos: (dolor, incomodi- } \\
\text { dad general). }\end{array}$ & $\begin{array}{l}- \text { Las correlaciones más altas entre } \\
\text { fatiga y somnolencia } \\
(r=0,772, P<0.001) \text {, depresión y } \\
\text { ansiedad }(r=0.858, P<0.001), \\
\text { y fatiga y apetito reducido }(r=0.414, P \\
<0.001) \text {. } \\
\text { - Los tres grupos de síntomas } \\
\text { predijeron independientemente la } \\
\text { QOL. }\end{array}$ \\
\hline
\end{tabular}




\begin{tabular}{|c|c|c|c|}
\hline $\begin{array}{c}\text { Autor, } \\
\text { año, } \\
\text { país }\end{array}$ & $\begin{array}{c}\text { Muestra: } \mathbf{n} ; \\
\text { edad; } \\
\% \text { hombres (H) }\end{array}$ & Objetivo & $\begin{array}{c}\text { Métodos } \\
\text { Estadísticos }\end{array}$ \\
\hline $\begin{array}{l}\text { Jumin } \\
\text { Park, } \\
\text { et al, } \\
2016, \\
\text { Estados } \\
\text { unidos, } \\
\text { China y } \\
\text { Taiwán }\end{array}$ & $\begin{array}{l}\cdot \text { EEUU: } \\
n=240 \\
63,5 \text { años } \\
59,2 \% \mathrm{H} \\
\cdot \text { Asia } \\
\mathrm{n}=240 \\
63,8 \text { años } \\
69,2 \% \mathrm{H}\end{array}$ & $\begin{array}{l}\text { Comparar los } \\
\text { síntomas y } \\
\text { grupo de } \\
\text { síntomas entre } \\
\text { pacientes de } \\
\text { Estados Unidos y } \\
\text { Asia con IC }\end{array}$ & $\begin{array}{l}\text { Algoritmo robusto de } \\
\text { máxima verosimilitud y } \\
\text { maximización de } \\
\text { expectativas (B. Muthén } \\
\text { y Shedden, 1999) }\end{array}$ \\
\hline
\end{tabular}

Clusters de Sintomas

Dos clúster de síntomas (EEUU Y

ASIA): (8 síntomas)

- Síntomas psicológicos (sentirse

deprimido, preocuparse y problemas cognitivos).

- Síntomas físicos: (edema, fatiga con necesidad de reposo, fatiga con baja energía, falta de aliento, dificultad para dormir).
Hallazgos clave

-Se realiza una comparación entre Estados Unidos y Asia, en donde la $p$ es significativa para los síntomas evaluados, se muestran diferencias entre dificultad para dormir y deterioro cognitivo. - Las personas de la cultura asiática tienden a expresar niveles más bajos de angustia emocional en comparación con aquellos con antecedentes culturales occidentales.

\begin{tabular}{|c|c|c|c|}
\hline $\begin{array}{l}\text { Moser } \\
\text { DK, } \\
\text { et al, } \\
2014, \\
\text { Estados } \\
\text { Unidos, } \\
\text { China- } \\
\text { Taiwán y } \\
\text { Países } \\
\text { bajos - } \\
\text { Suecia. }\end{array}$ & $\begin{array}{l}\cdot \text { EEUU: } \\
\mathrm{n}=240 \\
63,7 \text { años } \\
57,1 \% \mathrm{H} \\
\cdot \text { Asia* } \\
\mathrm{n}=240 \\
63,5 \text { años } \\
67,9 \% \mathrm{H} \\
\cdot \text { Europa: } \\
\mathrm{n}=240 \\
71,6 \text { años } \\
64,6 \% \mathrm{H}\end{array}$ & $\begin{array}{l}\text { Identificar y } \\
\text { comparar los } \\
\text { grupos de } \\
\text { síntomas en } \\
\text { pacientes con IC } \\
\text { en Estados } \\
\text { Unidos, Europa y } \\
\text { Asia. }\end{array}$ & $\begin{array}{l}\text { Análisis de } \\
\text { conglomerados, } \\
\text { aglomeración en racimo, } \\
\text { dendrogramas, } \\
\text { estadística } \\
\text { pseudo-F y estadística } \\
\text { cuadrada pseudo-T, } \\
\text { sistema euclidiano de } \\
\text { distancia }\end{array}$ \\
\hline
\end{tabular}

Dos clúster (8 síntomas)

- EEUU: Emocional (dificultad para

dormir,

preocuparse, sentirse deprimido y

problemas

cognitivos) y Físico (disnea, dificultad

para caminar).

- Asia: Emocional (preocuparse, sentirse deprimido,

problemas cognitivos) y Físico (disnea,

dificultad para

caminar, fatiga, dificultad para dormir).

- Europa: Emocional (preocuparse sentirse deprimido,

problemas cognitivos) y Físico (disnea, dificultad para caminar, fatiga).

\begin{tabular}{|c|c|c|c|c|}
\hline $\begin{array}{l}\text { Herr J, } \\
\text { et al, } \\
2014, \\
\text { Estados } \\
\text { Unidos. }\end{array}$ & $\begin{array}{l}\mathrm{n}=146 \\
56 \text { años } \\
62 \% \mathrm{H}\end{array}$ & $\begin{array}{l}\text { Identificar } \\
\text { grupos de } \\
\text { síntomas en } \\
\text { individuos con } \\
\text { IC y evaluar la } \\
\text { relación de } \\
\text { los grupos } \\
\text { identificados } \\
\text { con el estado } \\
\text { funcional. }\end{array}$ & $\begin{array}{l}\text { Análisis de correlación } \\
\text { con Pearson r., brechas } \\
\text { en la distribución de los } \\
\text { datos, múltiples picos y } \\
\text { valores atípicos. }\end{array}$ & $\begin{array}{l}\text { Tres clúster (10 síntomas) } \\
\text { - Comportamiento de la enfermedad } \\
\text { (ansiedad, síntomas de depresión, } \\
\text { somnolencias diurnas, disfunción } \\
\text { cognitiva, fatiga). } \\
\text { - Molestias gastrointestinales (apetito y } \\
\text { hambre). } \\
\text { - Malestar de la enfermedad (disnea, } \\
\text { edema y dolor). }\end{array}$ \\
\hline
\end{tabular}

\begin{tabular}{|c|c|c|c|}
\hline $\begin{array}{l}\text { Kyoung } \\
\text { SL, } \\
\text { et al, } \\
2010 \text {, } \\
\text { Georgia e } \\
\text { Indiana. }\end{array}$ & $\begin{array}{l}\mathrm{n}=331 \\
61 \text { años } \\
65 \% \mathrm{H}\end{array}$ & $\begin{array}{l}\text { Comparar los } \\
\text { grupos de } \\
\text { síntomas entre } \\
\text { hombres y } \\
\text { mujeres, las } \\
\text { diferencias } \\
\text { entre las } \\
\text { características } \\
\text { de los } \\
\text { pacientes y el } \\
\text { impacto } \\
\text { grupo de } \\
\text { síntomas en la } \\
\text { supervivencia de } \\
\text { eventos } \\
\text { cardiacos. }\end{array}$ & $\begin{array}{l}\text { Análisis de } \\
\text { conglomerados, Método } \\
\text { de Ward, análisis de } \\
\text { varianza o prueba } 2 \text { y } \\
\text { análisis de componentes } \\
\text { principales. }\end{array}$ \\
\hline
\end{tabular}

\section{Dos Clúster (8 síntomas)}

- Síntomas emocional/cognitivo (preocupación, problema cognitivos, sentirse deprimido)

- Síntomas físicos (disnea, fatiga con aumento de la necesidad de descanso fatiga/baja energía, y alteraciones del sueño).

EEUU y Asia, en Europa se

agrupó con la dificultad para dormir Se evidencia que el edema en IC es el único síntoma que no genera angustia en los pacientes a menos que sea extremo.

- La experiencia de los síntomas en las diferentes culturas es similar, sin embargo, se evidencia que e síntoma "Dificultad para dormir", en EEUU se encuentra en el grupo de síntomas emocionales y el Asia lo discriminan en el grupo de los síntomas físicos.

\section{- El 80\% de los participantes informó} limitaciones para realizar actividades de la vida diaria (caminar, subir escaleras, agacharse, arrodillarse). Limitación funcional $(\mathrm{F}=$

$17.68, \mathrm{P}=0.0005, \mathrm{R} 2=$

$0.591)$ modelo final de limitaciones funcionales $(F=35.95 \mathrm{p}=0.00005$ $\mathrm{R}=0.0578)$, movilidad: $(\mathrm{F}=8.96$. $\mathrm{P}=0.0005$. $\mathrm{R}=0.0305)$ Edad, modelo final de comorbilidad $(\mathrm{F}=20.68$. $\mathrm{P}=0.0005$. $\mathrm{R}=0.0276$ ) Comorbilidad.

- Las correlaciones más altas se observaron entre fatiga y somnolencia $(r=0,772, \mathrm{P}<0.001)$ depresión y ansiedad $(r=0.858$, $\mathrm{P}<0.001)$, y fatiga y apetito reducido $(r=0.414, P<0.001)$

- El edema y la mala calidad del sueño, se excluyeron del análisis de los grupos de síntomas.

- La puntuación total de la angustia de los síntomas en el grupo emocional/cognitivo, pero no en el grupo físico, fue un predictor independiente de supervivencia libre del evento cardíaco (tasa de riesgo, 1,18; IC del 95\%, 1,03 Y 1,37).

\begin{tabular}{|c|c|c|c|}
\hline $\begin{array}{l}\text { Hertzog } \\
\text { MA, } \\
\text { et al, } \\
\text { 2010, } \\
\text { Estados } \\
\text { Unidos }\end{array}$ & $\begin{array}{l}\mathrm{n}=139 \\
70,6(9,7) \text { años } \\
76 \% \mathrm{H}\end{array}$ & $\begin{array}{l}\text { Explorar el uso } \\
\text { del análisis } \\
\text { de grupos para } \\
\text { identificar } \\
\text { subgrupos de } \\
\text { pacientes con IC } \\
\text { cuyos síntomas } \\
\text { ayuden a guiar } \\
\text { el manejo } \\
\text { clínico. }\end{array}$ & $\begin{array}{l}\text { Análisis de } \\
\text { conglomerados } \\
\text { jerárquicos, distancias } \\
\text { euclidianas al cuadrado, } \\
\text { método de varianza } \\
\text { mínima de Ward. }\end{array}$ \\
\hline
\end{tabular}

\section{Tres clúster (14 síntomas)}

- Grupo 1: Baja carga de síntomas, reportan falta de aliento $55 \%$, fatiga $66 \%$

- Grupo 2: Mayor carga de síntomas, el $75 \%$ reportan disnea en las cuatro condiciones, fatiga, dificultad para dormir y olvido

- Grupo 3: Alta carga de síntomas. síntomas evaluados: mareo, edema, distensión, depresión, fatiga, disnea con actividad, disnea en reposo. Basados en la percepción de síntomas disnea en reposo, disnea con actividad disnea nocturna, disnea acostado, edema, distensión, fatiga, presión en el pecho, aleteo, tos, mareo, dificultad para dormir, olvido, depresión.

\begin{tabular}{|c|c|c|c|c|c|}
\hline $\begin{array}{l}\text { Song EK, } \\
\text { et al, } \\
2010 \text {, } \\
\text { Corea del } \\
\text { sur }\end{array}$ & $\begin{array}{l}n=421 \\
62 \text { años } \\
60,3 \% H\end{array}$ & $\begin{array}{l}\text { Identificar el } \\
\text { grupo de } \\
\text { síntomas físicos } \\
\text { en IC y su } \\
\text { relación con la } \\
\text { supervivencia } \\
\text { libre de } \\
\text { hospitalizaciones }\end{array}$ & $\begin{array}{l}\text { Enfoque de agrupación } \\
\text { jerárquica aglomerada } \\
\text { con el método de Ward y } \\
\text { Cuadrado Euclidiano de } \\
\text { distancias. }\end{array}$ & $\begin{array}{l}\text { Dos clúster (6 síntomas) } \\
\text { - Síntomas disneicos (disnea, levantarse } \\
\text { sin respiración por la noche, disnea } \\
\text { acostado). } \\
\text { - Síntomas fatigados (dificultad para } \\
\text { dormir, apetito, falta de energía). }\end{array}$ & $\begin{array}{l}\text { - Los pacientes que experimentaron } \\
\text { más angustia en el grupo de } \\
\text { fatiga tuvieron un riesgo de } 50 \% \text { de } \\
\text { rehospitalización. } \\
\text { - El edema de tobillos no se agrupó en } \\
\text { clúster. }\end{array}$ \\
\hline
\end{tabular}

Tabla elaborada propia de los autores. *Asia: China y Taiwán QOL: calidad de vida IC: insuficiencia cardiaca NYHA:Clase funcional. 


\section{Objetivos de los estudios}

Los estudios plantearon diferentes objetivos en los cuales los participantes comparten características clínicas como clase funcional III/IV y algunos síntomas comunes como fatiga, disnea y depresión, así mismo los grupos de síntomas fueron evaluados desde las esferas físico y mental 11,19,20,22,24. En algunos estudios el objetivo planteado fue identificar los clúster de síntomas ${ }^{10}$, en otros se establecieron los clúster en asociación con variables sociodemográficas ${ }^{25}$ y en otros, adicionalmente, se relacionaron los grupos de síntomas con variables como: calidad de vida, estado funcional, supervivencia libre de eventos, mortalidad y supervivencia libre de hospitalizaciones ${ }^{10,12,28,20,21}$

\section{Características sociodemográficas de la población}

Los 11 estudios que fueron seleccionados de acuerdo a los criterios de inclusión y evaluación realizada, reúnen en total 7045 pacientes con diagnóstico de insuficiencia cardiaca, de estos se tomaron solo los pacientes con clase funcional III/ IV. En cuanto al género, en las muestras de todos los estudios recopilados se ha reportado más del 50\% de hombres, así mismo, la edad de los pacientes en los estudios reportan las medidas de tendencia central, tales como la media, un análisis de estos valores muestra cómo se mueve en rango mínimo de 56 años y máximo de 83 años aproximadamente; otras variables sociodemográficas reportan tan solo un $5 \%$ como son: la raza, ocupación y el estado civil, ver tabla $2^{10,11,12,18,19,20,21,22,23,24,25}$.

\section{Medidas del estudio}

En el grupo de artículos seleccionados se identificaron, diversos instrumentos utilizados para la medición de los síntomas en los pacientes, el de mayor uso (54\%), fue el Índice de comorbilidad de Charlson, reportado en seis artículos. Este instrumento fue utilizado para explorar la mortalidad a largo plazo partiendo de las comorbilidades en los pacientes; derivando que las comorbilidades más frecuentes descritas por los pacientes fueron la diabetes mellitus, la enfermedad renal, la enfermedad pulmonar crónica y la enfermedad de tejido conectivo. Se ha considerado que las comorbilidades pueden influir en los síntomas asociados a la falla cardiaca y que son un factor limitante para el individuo, no obstante, no limita su capacidad de realizar actividades físicas ${ }^{10,12,18,21,22,23}$.

Otra de las herramientas utilizadas en los estudios correspondió el cuestionario de Minnesota living with heart failure Questionnaire (MLHFQ), con (45\%) reportados en cinco estudios 11,19,20,22,24 .

Dos estudios utilizaron la disnea con subescala de síntomas y limitaciones cardiovasculares (CLASP), la cual consta de cinco (5) preguntas que evaluaban la percepción de escasez de aliento durante las 2 semanas anteriores. Los ítems valoran la frecuencia, severidad e interferencia con las actividades; determinándose como leve, moderado o severo ( 5 a 7 puntos = leve; 8 a 10 puntos = moderado; 11 a 14 puntos = severo). La sumatoria de estos ítems permitió calcular la puntuación de la disnea, teniendo en cuenta que la puntuación más alta indicaba la disnea más severa ${ }^{12,18}$.

Otros estudios aplicaron la escala de Ansiedad hospitalaria y la escala de depresión (HADS), para la evaluación de la ansiedad y la depresión; cada subescala se componía de siete elementos en una escala Likert de 4 puntos desde ausencia (nivel inferior $=0$ ) hasta ocurrencia la mayor parte del tiempo (nivel superior $=3)^{12,18}$.

\section{Análisis Estadístico}

Los métodos de análisis estadísticos reportados, incluyen desde medidas de frecuencias o análisis descriptivos ${ }^{10,18,22,23,25}$ hasta medidas de análisis complejos para la determinación de clúster. La mayoría de los estudios utilizaron técnicas de análisis por conglomerados ${ }^{19,20,21,22,23,25}$, otros utilizaron el análisis de correlación con Pearson $r^{10,12,18,20}$. 


\section{Síntesis de los clúster recopilados en los estudios}

Cada uno de los estudios que fueron incluidos en la revisión sistemática, está sintetizado en la tabla 3. En cada uno de los clúster se halló la frecuencia en la que se presentaron los síntomas y se organizaron de mayor a menor frecuencia, así se logró establecer que el síntoma de mayor frecuencia fue la depresión reportado en nueve estudios (82\%), fue relacionado en seis clúster de síntomas (Comportamiento de la enfermedad, síntomas emocionales, síntomas angustiosos, síntomas afectivos, síntomas psicológicos, Grupo 3: Alta carga de síntomas ${ }^{10,11,12,18,19,22,23,24,25}$ ), así mismo, la dificultad para dormir fue reportados en ocho estudios (72\%) relacionada en tres clúster de síntomas (síntomas emocionales, síntomas físicos y síntomas fatigado) $11,19,20,21,22,23,24,25$. La disnea fue reportada en siete estudios (63\%) y se encuentra presente en tres clúster de síntomas (Malestar de la enfermedad, síntomas físicos y síntomas angustiosos) $10,12,18,19,20,22,23$, finalmente el deterioro cognitivo y la fatiga fueron reportados con una frecuencia de seis estudios (54\%). El deterioro cognitivo se presentó en tres clúster de síntomas (Comportamiento de la

En cada uno de los clúster se halló la frecuencia en la que se presentaron los síntomas y se organizaron de mayor a menor frecuencia, así se logró establecer que el síntoma de mayor frecuencia fue la depresión reportado en nueve estudios ( $82 \%)$, fue relacionado en seis clúster de síntomas (Comportamiento de la enfermedad, síntomas emocionales, síntomas angustiosos, síntomas afectivos, síntomas psicológicos, Grupo 3: Alta carga de síntomas $10,11,12,18,19,22,23,24,25)$, enfermedad, síntomas emocionales y síntomas psicológicos) $)^{11,12,18,19,22,24}$, la fatiga se muestra en seis clúster de síntomas (Comportamiento de la enfermedad, síntomas físicos y síntomas de descondicionamiento, Grupo 1 baja carga de síntomas, Grupo 2 mayor carga de síntomas, Grupo 3 alta carga de síntomas) $)^{10,12,18,19,20,25}$.

De una manera diferente como muestra Huang TY et $\mathrm{al}^{20}$, en su estudio; se identificaron tres grupos de síntomas en función de la gravedad de los síntomas físicos evaluados: disnea, fatiga, edema, insomnio, anorexia y mala memoria. Estos autores nombraron un grupo de síntomas no severos (todos los síntomas se calificaron con baja gravedad), un grupo de síntomas de gravedad típico (alto nivel de gravedad para disnea y fatiga, bajo nivel de gravedad para edema y nivel moderado de gravedad para todos los demás síntomas) y otro grupo de síntomas de gravedad atípica (bajo nivel de gravedad para disnea y fatiga, alto nivel de gravedad para edema y nivel moderado de gravedad para todos los demás síntomas). Sobre la base de los resultados de este estudio, es importante evaluar a los pacientes con insuficiencia cardiaca con síntomas físicos atípicos cómo el edema de las piernas, el insomnio, la anorexia y la falta de memoria, estos evidencian una tendencia límite $(p=0,061)$ lo que mostró un aumento en el riesgo de eventos cardíacos comparado con los otros grupos, el seguimiento se realizó en un periodo de 12 meses $^{20}$.

En esta misma tendencia de nombrar los grupos de síntomas de una manera particular, como muestra Hertzog, M.A. et al ${ }^{25}$; optaron por llamar los clúster, como Clúster de síntomas 1, 2,3, agrupando en ellos 15 síntomas. Bajo esta manera de llamar los clúster se agruparon los síntomas para el clúster 1 como aquellos que establecían una baja carga de síntomas, incluyendo dentro de él la fatiga y falta de aliento. Para el grupo del clúster 2 se estableció la mayor carga de siete síntomas (fatiga, dificultad para dormir, olvido, disnea en sus cuatro presentaciones) y por último el clúster 3 que incluía una alta carga de síntomas, tuvo seis síntomas (fatiga, depresión, edema, disnea con activa y disnea en reposo). Este último clúster fue el más predominante en los pacientes con clasificación III o IV de la NYHA (78,9\%). 
Los estudios seleccionados en la revisión sistemática han permitido identificar cuáles son los clústers de síntomas que se presentan con mayor frecuencia en los estadios III o IV de pacientes con insuficiencia cardiaca. Este resumen de síntomas resulta un aporte importante para el personal de enfermería quien puede realizar una mejor intervención y para el personal médico quien puede realizar una prescripción más ajustada, con el propósito de disminuir el sufrimiento en el paciente y su familia ${ }^{8,16}$
Este resumen de síntomas resulta un aporte importante para el personal de enfermería quien puede realizar una mejor intervención y para el personal médico quien puede realizar una prescripción más ajustada, con el propósito de disminuir el sufrimiento en el paciente y su familia $^{8,16}$

VOSviewer

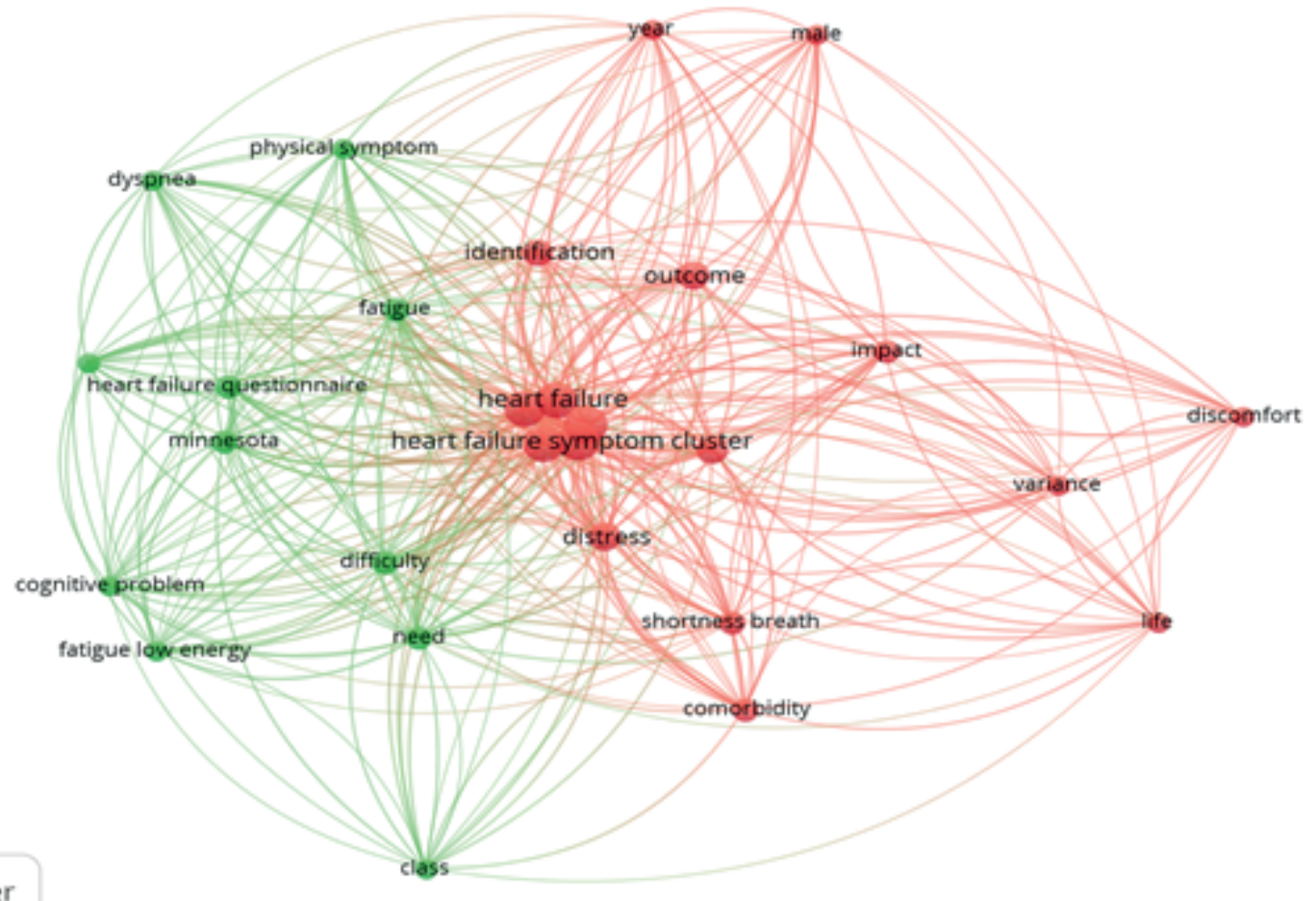

Figura 2. Representación gráfica de co-ocurrencia de palabras en los resúmenes de los estudios seleccionados

Para evaluar de manera cualitativa el contenido de los resúmenes de los artículos, se realizó un análisis con el software VOSviewer, el cual, a través de mapas bibliométricos, emite una representación gráfica de la frecuencia de las palabras y sus relaciones de acuerdo con la ocurrencia en que se presentan, luego de depuración de palabras similares bajo una misma denominación ${ }^{27}$. La fuente para estos análisis fueron los títulos y resúmenes de los artículos incluidos en esta revisión sistemática. Los análisis incluyeron en las redes de interconexión las palabras que tuviesen una frecuencia de por lo menos tres veces.

De acuerdo con lo anterior, en la Figura 2 se identificaron dos agrupaciones y colores. La primera de ellas (Color Rojo) mostró aquellas palabras que hacían referencia a la metodología y análisis realizado dentro de los estudios, con palabras como (impacto, resultado, varianza). La segunda agrupación (Color Verde) incluye palabras que refieren diferentes tipos de síntomas (fatiga baja energía, problemas cognitivos, disnea, dificultad para dormir), evaluados dentro de los estudios, por esta razón, incluye el cuestionario de Minnesota Living With Heart Failure (MLHFQ), verificando su utilización en la mayoría de los estudios. Los dos nodos con mayor tamaño, ubicados en la parte central del gráfico: insuficiencia cardiaca y clúster de síntomas, demuestran que las palabras y las temáticas desarrolladas en los artículos, giraron en torno a ellos, lo que sirve para evidenciar que los artículos analizados cumplían con los criterios para ser incluidos en la presente revisión. 
Tabla 3. Síntesis de los clúster de síntomas recuperados de los estudios en insuficiencia cardiaca avanzada.

\begin{tabular}{|c|c|c|c|c|c|c|c|c|c|c|c|c|c|c|c|c|c|c|c|c|c|c|}
\hline |вłO| & in & $m$ & - & 0 & in & $\nabla$ & $\nabla$ & $m$ & N & $\nabla$ & $m$ & N & - & $\nabla$ & $m$ & $m$ & $m$ & N & $\sim$ & $\bullet$ & N & $\stackrel{m}{\sim}$ \\
\hline оәлеW & & & & & & & & & & & & & & & & & & & & $x$ & & - \\
\hline е!хәлочн & & & & $\times$ & & & & & & & & & & & & & & & & & & $r$ \\
\hline seəsneN & & & & & & & & & & $x$ & & & & & & & & & & & & - \\
\hline osodəy uә eəus!a & & & & & & & & & & & & & & & & & & & $x$ & $x$ & & $n$ \\
\hline 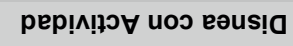 & & & & & & & & & & & & & & & & & & & $x$ & $x$ & & $N$ \\
\hline ә૫ગоน е| uә еәus! & & & & & & & & & & & & $x$ & & & & & & & $x$ & & & $n$ \\
\hline opełsose eәus!a & & & & & & & & & & & & $x$ & & & & & & & $x$ & & & N \\
\hline 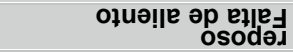 & & & & & $x$ & & & & & & & & & & & & & & & & $x$ & $N$ \\
\hline 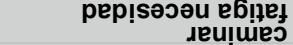 & & & & & $x$ & & $x$ & & & & & & & & & & & & & & & N \\
\hline e.jed pełןกग!!ด & & & & & & $x$ & & $x$ & & & & & & & & & & & & & & N \\
\hline ouın!p в!эuәрuسos & $x$ & & & & & & & & & $x$ & & & & & & & & & & & & $N$ \\
\hline 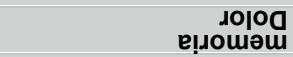 & & $x$ & & & & & & & & & & & $\times$ & & & & & & & & & $N$ \\
\hline ереш о ор!^ıо & & & & $\times$ & & & & & & & & & & & & & & & $x$ & & & N \\
\hline 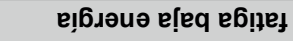 & & & & & $x$ & & $x$ & & & & $x$ & & & & & & & & & & & $m$ \\
\hline ol!?əd $\forall$ & & & $x$ & & & & & & & $x$ & $x$ & & & & & & & & & & & $m$ \\
\hline uọ!oednooə.dd & & & & & & & & & & & & & & $x$ & $x$ & $x$ & & & & & & $m$ \\
\hline рерәџฺsu & $x$ & & & & & & & & & & & & & & & & $x$ & $x$ & & & & $m$ \\
\hline ешәр $\exists$ & & $x$ & & $x$ & $x$ & & & & & & & & & & & & & & & $x$ & & $\theta$ \\
\hline ом!ฺ!ибог одо!ләңәа & $x$ & & & & & & & & & & & & & $x$ & $x$ & $x$ & & & & & & $\theta$ \\
\hline uọ!sə.ıdəa & $x$ & & & & & & & & & & & & & $x$ & $x$ & $x$ & $x$ & $x$ & & $x$ & & $r$ \\
\hline eəus!a & & $\times$ & & $x$ & & $x$ & $x$ & $x$ & $x$ & & & & & & & & $x$ & & & & & $\Lambda$ \\
\hline ג!usop esed peł|nગ!!! & & & & $\times$ & $\times$ & $x$ & $x$ & & $x$ & & $x$ & & & $x$ & & & & & $x$ & & & $\infty$ \\
\hline e6!pe & $x$ & & & $\times$ & & $x$ & & $x$ & & $x$ & & & & & & & & & $x$ & $x$ & $x$ & $\infty$ \\
\hline 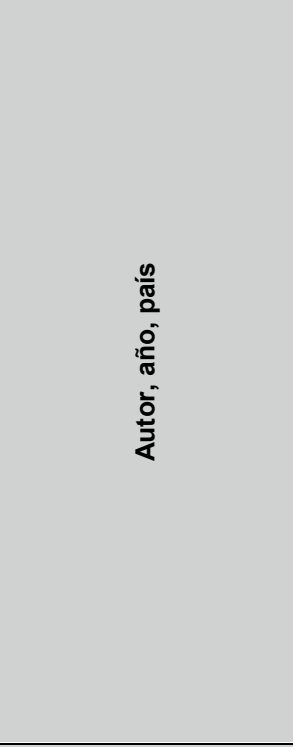 & & 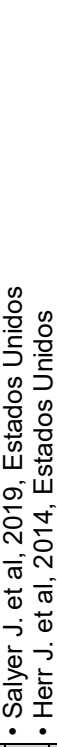 & & 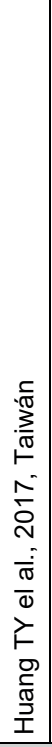 & 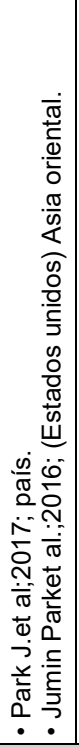 & 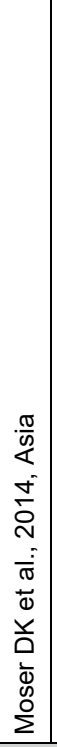 & 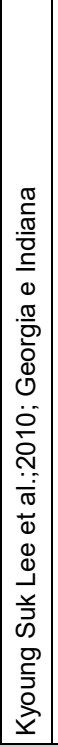 & 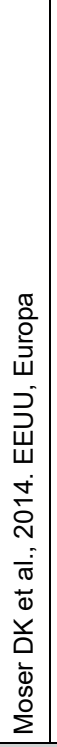 & 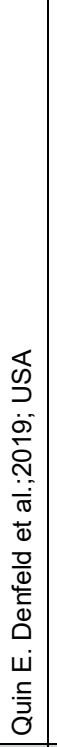 & 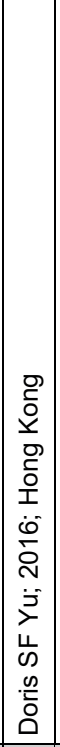 & 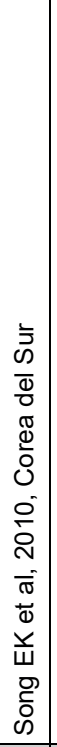 & 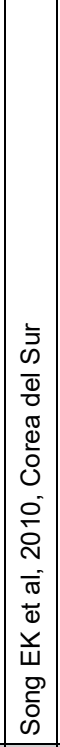 & 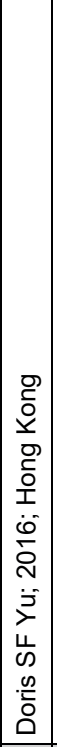 & 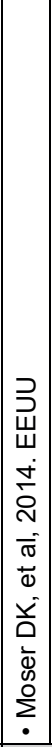 & 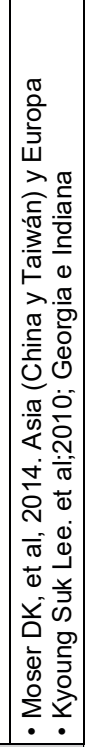 & 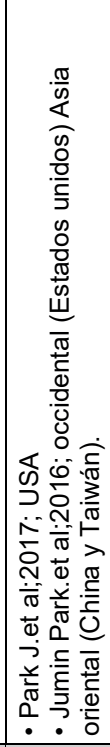 & 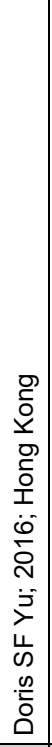 & 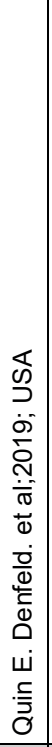 & & 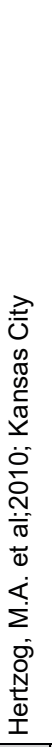 & & \\
\hline 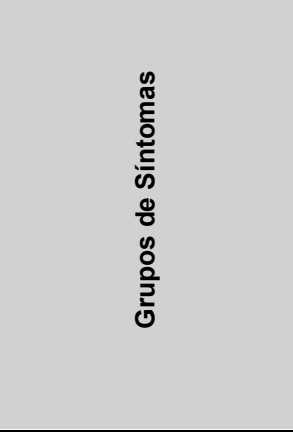 & 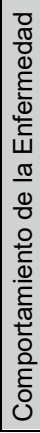 & 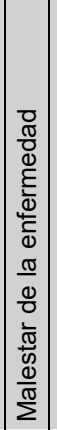 & 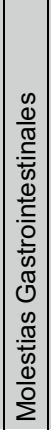 & & & 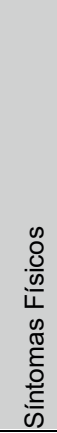 & & & & 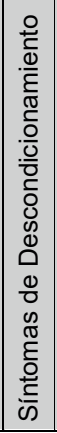 & 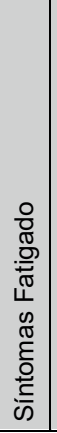 & 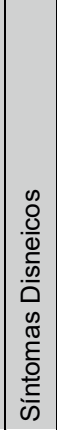 & 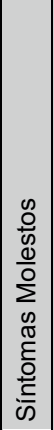 & & 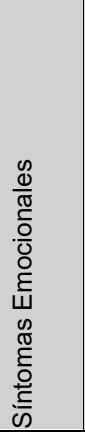 & $\begin{array}{l}00 \\
\frac{0}{0} \\
.0 \\
0 \\
\frac{0}{\infty} \\
0 \\
0 \\
0 \\
\tilde{0} \\
0 \\
0 \\
0 \\
0\end{array}$ & 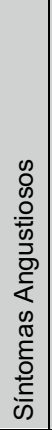 & 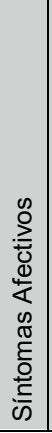 & 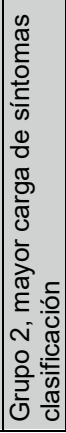 & 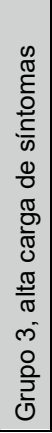 & 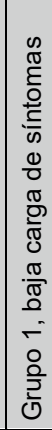 & $\begin{array}{l}\frac{1}{\leqslant} \\
\text { O } \\
\text { L }\end{array}$ \\
\hline
\end{tabular}




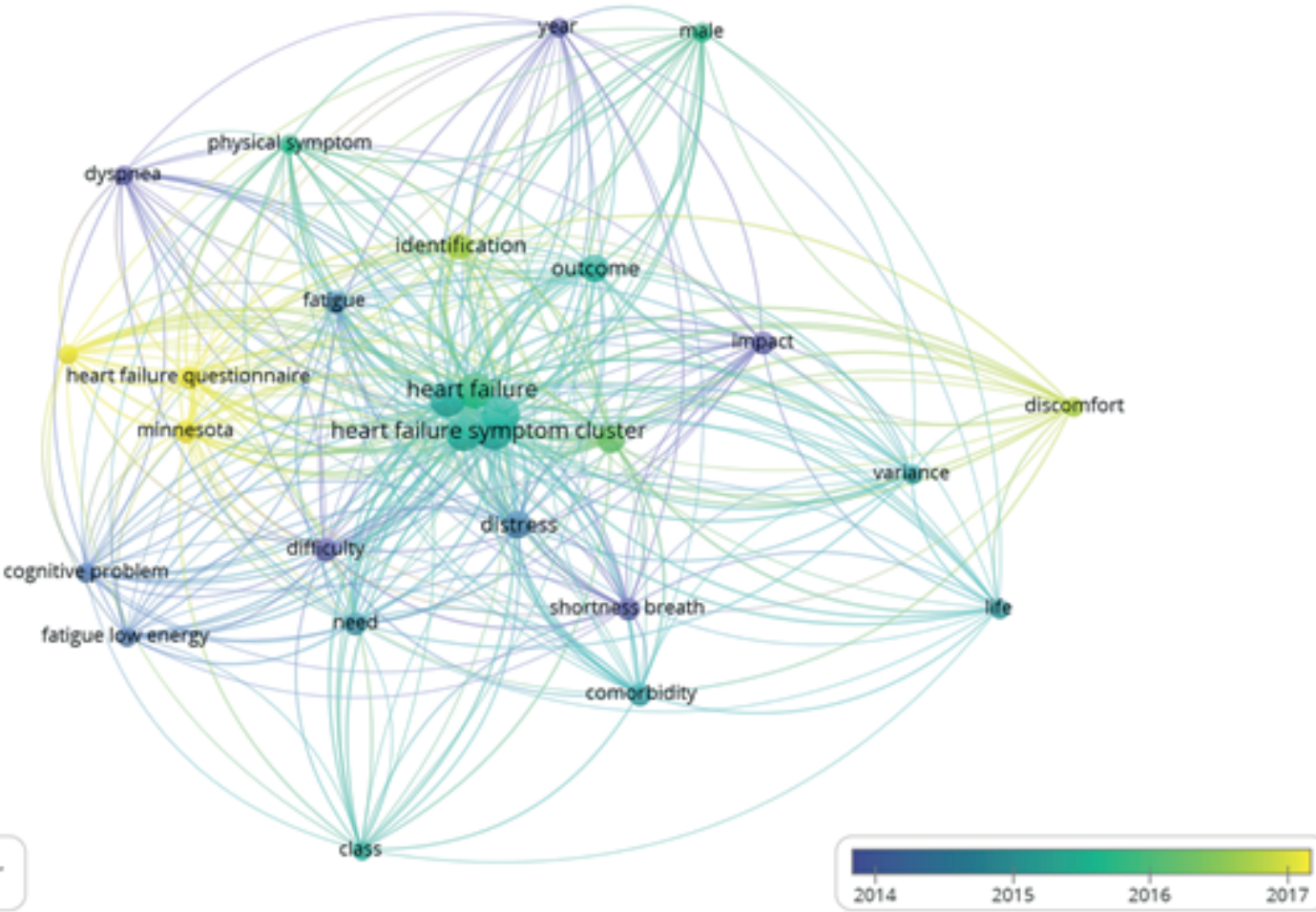

vosviewer

\section{Figura 3. Co-ocurrencia de palabras en los resúmenes de los estudios seleccionados según su fecha de publicación.}

Continuando con este análisis visual, la Figura No 3, en su barra de tiempo de la parte inferior derecha, mostró a través de los colores, la evolución del uso de las palabras a través de los años en que fueron publicados los artículos. Esta gráfica demostró, cómo en un primer momento (color azul) los estudios referían términos que hicieron alusión a la sintomatología: angustia y fatiga, y a los impactos y dificultades en los pacientes. Un segundo grupo (color azul verdoso), mostró los términos de falla cardiaca y clúster de síntomas en insuficiencia cardiaca, que además estando ubicados en el centro de la gráfica, ratificó la existencia de una gran conexión de las palabras con estos temas centrales. Finalmente las palabras de los artículos de más reciente publicación (color amarillo), mostraron el uso de los cuestionarios para evaluar falla cardiaca en especial el cuestionario de Minnesota Living With Heart Failure (MLHFQ) para identificación de los síntomas.

Es importante resaltar que los resultados de este estudio proporcionan información sobre los grupos de síntomas en pacientes con insuficiencia cardiaca avanzada, sin embargo, se reconocen las siguientes limitaciones: primero en esta revisión se incluyeron dos estudios del mismo autor con diferente año de publicación, en donde se describe la misma población, y utilizaron los mismos clúster de síntomas, sólo que su objetivo consiste en analizar diferentes relaciones en eventos como calidad de vida y estado funcional ${ }^{12,18}$, segundo las variables sociodemográficas tales como: raza, estado civil y ocupación, no fueron detalladas en todos los estudios, sólo en el $5 \%$ se discriminaron, por lo que no se logra el análisis de esos resultados y tercero por otra parte a pesar que el objetivo general estaba centrado en identificar los clúster de síntomas que se establecieron, un resultado interesante era contar con la frecuencia de estos síntomas en cada una de las poblaciones, con el fin de realizar un análisis en el cual se pudiera explorar si existían supragrupos. 


\section{Discusión}

Esta revisión sistemática permitió la identificación de los clúster de síntomas en insuficiencia cardiaca desde las dimensiones física y psicológica, los autores han planteado diferentes denominaciones al etiquetar cada grupo de síntomas y utilizaron diferentes criterios para la inclusión de cada síntoma en los clústers identificados. Durante la revisión se encontró que el diseño del estudio más representativo es de tipo corte transversal, las características de la muestra evidencian que, en cuanto al género, más del $50 \%$ son hombres, así mismo, la edad de los pacientes en los estudios oscila en un rango mínimo de 56 años y máximo de 83 años. Por otra parte las variables sociodemográficas como: la raza, ocupación y el estado civil, se discriminan en los estudios tan sólo en un 5\%, por lo tanto, no fue factible hacer una discusión que permita establecer si estas variables afectan los grupos de síntomas y así poder determinar si se deben tener en cuenta al momento de realizar el abordaje de los pacientes con insuficiencia cardiaca avanzada. Los análisis estadísticos son variados, lo que hace que sea difícil comparar resultados entre estudios y generalizar los hallazgos, así mismo, los estudios plantearon objetivos que relacionaron los grupos de síntomas con variables como: calidad de vida, estado funcional, supervivencia libre de eventos, mortalidad y supervivencia libre de hospitalizaciones, con el fin de desarrollar intervenciones para mejorar calidad de vida y evitar el sufrimiento.

El síntoma edema fue reportado en el $80 \%$ de los estudios, en uno de ellos llama la atención que es el único síntoma que no generó angustia en los pacientes a menos que sea extremo ${ }^{22}$. El síntoma dolor, en esta revisión, se presentó con una frecuencia de tres estudios con un $27 \%$ y se encuentra en dos clúster denominados (malestar general y síntomas molestos), así mismo, el clúster de malestar general tiene tres síntomas relacionados entre sí, (dolor, edema y disnea $)^{12}$, los causales son causales de una descompensación cardiaca o una fase terminal de la enfermedad, donde el paciente empieza a experimentar síntomas refractarios, molestos, lo cual ocasiona un aumento de las necesidades paliativas.

La Organización Mundial de la Salud en el año 2018, define los cuidados paliativos como "la prevención y el alivio del sufrimiento de pacientesadultosy pediátricos y sus familias que enfrentan los problemas asociados con enfermedades potencialmente mortales. Estos problemas incluyen sufrimiento Biopsicosocial y espiritual de los pacientes y miembros de la familia ${ }^{28}$, teniendo en cuenta los pacientes que son candidatos a recibir los cuidados paliativos, así mismo la OMS "considera que las enfermedades crónicas como enfermedades cardiovasculares, cáncer, enfermedades respiratorias crónicas, SIDA, diabetes mellitus y otras pueden requerir asistencia paliativa"29.

\begin{abstract}
Define los cuidados paliativos como “la prevención y el alivio del sufrimiento de pacientes adultos y pediátricos y sus familias que enfrentan los problemas asociados con enfermedades potencialmente mortales. Estos problemas incluyen sufrimiento Biopsicosocial y espiritual de los pacientes y miembros de la familia $" 28$
\end{abstract}


Los pacientes con insuficiencia cardiaca avanzada, pueden presentar cambios en su sintomatología hasta llegar a empeorar a pesar de estar recibiendo tratamientos óptimos, una posibilidad de manejo son considerados los cuidados paliativos cuyo objetivo se centra en el control de sus síntomas y generar planes de cuidado en el manejo de los síntomas que pueden ser abordados de forma individual $o$ grupales, permitiendo el alivio al sufrimiento ${ }^{8,16}$; resulta importante reconocer que al tratar un síntoma incluido en un clúster se puede llegar a mejorar los demás síntomas que estén en su mismo grupo, brindando mejores beneficios en el paciente ${ }^{12}$.
Los pacientes con insuficiencia cardiaca avanzada, pueden presentar cambios en su sintomatología hasta llegar a empeorar a pesar de estar recibiendo tratamientos óptimos, una posibilidad de manejo son considerados los cuidados paliativos cuyo objetivo se centra en el control de sus síntomas y generar planes de cuidado en el manejo de los síntomas que pueden ser abordados de forma individual o grupales, permitiendo el alivio al sufrimiento ${ }^{8,16}$

\section{Conclusiones}

La agrupación de los síntomas para paciente con insuficiencia cardiaca avanzada corresponde a diferentes conceptos, algunos de ellos derivados de la presentación específica de los síntomas y otras de diferentes formas de medir la gravedad de los síntomas en los pacientes, así mismo los estudios presentan diversas características tanto en sus criterios de inclusión y exclusión, como su forma de recolección de datos, lo que hace evidente que no existe uniformidad en los resultados encontrados, por lo que no se podrían generalizar los hallazgos entre los estudios. En la mayoría de los estudios se utilizan instrumentos estandarizados, para medir los síntomas o para recopilar los síntomas que presentan los pacientes. Los análisis estadísticos reportados incluyen desde medidas de frecuencias o análisis descriptivos, hasta análisis complejos para la determinación de clústers, por lo cual esta diversidad no permite, hacer una comparación entre la forma en que se hallaron los clústers, lo que muestra que para futuras investigaciones sería un reto estandarizar un instrumento para la determinación de los clústers. Así mismo los estudios reportan diferentes objetivos complementarios tales como: supervivencia, estado funcional y calidad de vida, plantear diferentes objetivos, permite crear un mejor panorama de abordaje terapéutico en donde la severidad de los síntomas evidencia la progresión de la enfermedad en los pacientes, lo que conlleva a individualizar los tratamientos y paliar los síntomas.

Para finalizar se considera que identificar los clústers de síntomas en pacientes con insuficiencia cardiaca avanzada, permitirá realizar un abordaje integral de la carga de síntomas del paciente con necesidades paliativas y así mismo enfrentar el reto de aliviar el sufrimiento.

Financiación: Esta revisión no contó con ningún tipo de financiación.

Conflicto de Intereses: Los autores declaran no tener ningún conflicto de intereses. 


\section{Referencias}

1. Xavier SD, Ferretti-Rebustini RE. Características clínicas de la Insuficiencia Cardíaca asociadas a la dependencia funcional en la admisión de los adultos mayores hospitalizados. Revista Latino-Americana de Enfermagem. 2019;27. http://dx.doi.org/10.1590/1518-8345.2869-3137.

2. Fundación Española del Corazón. Insuficiencia Cardiaca. [Online].; 2017 [cited 2020 Mayo 24]. Available: https:/fundaciondelcorazon.com/informacion-para-pacientes/ enfermedades-cardiovasculares/insuficiencia-cardiaca.html

3. Haedtke CA, Moser DK, Pressler SJ, Chung ML, Wingate S, Goodlin SJ. Influence of depression and gender on symptom burden among patients with advanced heart failure: Insight from the pain assessment, incidence and nature in heart failure study. Heart \& Lung. 2019;48(3):201-207. https://doi.org/10.1016/j.hrtlng.2019.02.002

4. Yu DS, Li PW, Chong SO. Symptom cluster among patients with advanced heart failure: a review of its manifestations and impacts on health outcomes. Current opinion in supportive and palliative care. 2018;12(1):16-24. https://doi.org/10.1097/SPC.0000000000000316

5. Grant, L.; Downing, J.; Luyirika, E.; Murphy, M.; Namukwaya, L.; Kiyange, F.; et al. Integrating palliative care into national health systems in Africa: A multi-country intervention study. J. Glob. Health. 2017;7:010419. https://doi.org/10.7189/jogh.07.010419

6. Gómez-Mesa JE, Saldarriaga C, Jurado AM, Mariño A, Rivera A, Herrera Á, et al. Consenso colombiano de falla cardíaca avanzada: capítulo de Falla Cardíaca, Trasplante Cardíaco e Hipertensión Pulmonar de la Sociedad Colombiana de Cardiología y Cirugía Cardiovascular. Revista Colombiana de Cardiología. 2019;26:3-24. https://doi.org/10.1016/j.rccar.2019.06.001

7. Colombia. Ministerio de Salud y Protección Social. Guía de práctica clínica para la prevención, diagnóstico, tratamiento y rehabilitación de la falla cardíaca en población mayor de 18 años clasificación B, C y D. Guía para profesionales de la salud . 2016. Guía no. 53. Disponible en: http://gpc.minsalud.gov.co/gpc_sites/Repositorio/Conv_637/GPC_falla_ cardiaca/GPC\%20Falla\%20Cardiaca\%20Profesionales\%20No\%2053.pdf

8. Sleeman KE, de Brito M, Etkind S, Nkhoma K, Guo P, Higginson IJ, et al. The escalating global burden of serious health-related suffering: projections to 2060 by world regions, age groups, and health conditions. The Lancet Global Health. 2019;7(7):e883-92. https://doi.org/10.1016/S2214-109X(19)30172-X

9. Lowey SE. Palliative Care in the management of patients with advanced heart failure. InHeart Failure: From Research to Clinical Practice 2017 (pp. 295-311). Springer, Cham. https://doi.org/10.1007/5584_2017_115

10.Yu DS, Chan HY, Leung DY, Hui E, Sit JW. Symptom clusters and quality of life among patients with advanced heart failure. Journal of geriatric cardiology: JGC. 2016;13(5):408-14. https://doi.org/10.11909/j.issn.1671-5411.2016.05.014

11.Park J, Moser DK, Griffith K, Harring JR, Johantgen M. Exploring symptom clusters in people with heart failure. Clinical Nursing Research. 2019;28(2):165-81. https://doi.org/10.1177/1054773817729606

12.Salyer J, Flattery M, Lyon DE. Heart failure symptom clusters and quality of life. Heart \& Lung. 2019;48(5):366-72. https://doi.org/10.1016/j.hrtlng.2019.05.016

13.Carrillo-González GM. Los grupos de síntomas en personas con cáncer: una revisión integrativa. Aquichan. 2017;17(3):257-69. https://doi.org/10.5294/aqui.2017.17.3.3

14.Díaz CC. Percepción de Los síntomas desagradables por la persona hemodializada. Análisis a la luz de la teoría de Elizabeth Lenz. CURAE. 2019;2(1):63-79.

https://doi.org/10.26495/curae.v2i1.1113 
15.Jaramillo DMG, Muñoz CO, Nuvan ANS, Benavides FPA. Revisión sistemática para el abordaje de síntomas desagradables gastrointestinales en cuidados paliativos. Rev Cuid. 2019;10(1):e615. http://dx.doi.org/10.15649/cuidarte.v10i1.615

16.González-Robledo G, León J, Buitrago AF, Carvajalino S , Abril D, González V, et al. Cuidado paliativo en falla cardiaca. Rev Colomb Cardiol.2017;24(3):286-296. http://dx.doi.org/10.1016/j.rccar.2016.10.049

17. Hutton B, Catala-Lopez F, Moher D. The PRISMA statement extension for systematic reviews incorporating network meta-analysis: PRISMA-NMA. Med Clin (Barc). 2016;147(6):262-6. http://dx.doi.org/10.1016/j.medcli.2016.02.025

18. Herr JK, Salyer J, Flattery M, Goodloe L, Lyon DE, Kabban CS, Clement DG. Heart failure symptom clusters and functional status-a cross-sectional study. Journal of advanced nursing. 2015 Jun;71(6):1274-87. https://doi.org/10.1111/jan.12596

19.Moser DK, Lee KS, Wu JR, Mudd-Martin G, Jaarsma T, Huang TY, et al. Identification of symptom clusters among patients with heart failure: An international observational study. International Journal of Nursing Studies. 2014;51(10):1366-72.

https://doi.org/10.1016/j.ijnurstu.2014.02.004

20.Huang TY, Moser DK, Hwang SL. Identification, associated factors, and prognosis of symptom clusters in Taiwanese patients with heart failure. Journal of Nursing Research. 2018;26(1):60-7. https://doi.org/10.1097/JNR.0000000000000199

21.Song EK, Moser DK, Rayens MK, Lennie TA. Symptom clusters predict event-free survival in patients with heart failure. The Journal of cardiovascular nursing. 2010 Jul;25(4):284.

https://doi.org/10.1097/JCN.0b013e3181cfbcbb

22. Lee KS, Song EK, Lennie TA, Frazier SK, Chung ML, Heo S, et al. Symptom clusters in men and women with heart failure and their impact on cardiac event-free survival. Journal of Cardiovascular Nursing. 2010;25(4):263-72. https://doi.org/10.1097/JCN.0b013e3181cfbb88

23.Denfeld QE, Bidwell JT, Gelow JM, Mudd JO, Chien CV, Hiatt SO, Lee CS. Cross-classification of physical and affective symptom clusters and 180-day event-free survival in moderate to advanced heart failure. Heart \& Lung. 2020;49(2):151-7.

https://doi.org/10.1016/j.hrtlng.2019.11.004

24.Park J, Johantgen ME. A cross-cultural comparison of symptom reporting and symptom clusters in heart failure. Journal of Transcultural Nursing. 2017;28(4):372-80.

https://doi.org/10.1177/1043659616651673

25.Hertzog MA, PozehI B, Duncan K. Cluster analysis of symptom occurrence to identify subgroups of heart failure patients: a pilot study. Journal of Cardiovascular Nursing. 2010;25(4):273-83. https://doi.org/10.1097/JCN.0b013e3181cfbb6c

26.Cartes-Velasquez R, Moraga J. Pautas de chequeo, parte III: STROBE y ARRIVE. Revista chilena de cirugía. 2016;68(5):394-9. https://doi.org/10.1016/j.rchic.2015.12.003

27.Gálvez C. Visualización de las principales líneas de investigación en salud pública: un análisis basado en mapas bibliométricos aplicados a la Revista Española de Salud Pública (20062015). Rev Esp Salud Pública. 2016;90:e1-e10.

Disponible en: https://www.redalyc.org/pdf/170/17043728048.pdf

28.World Health Organization. Integrating palliative care and symptom relief into primary health care: A WHO guide for planners, implementers and managers. 2018. Retrieved from: https://www.who.int/publications/i/item/integrating-palliative-care-and-symptom-reliefinto-primary-health-care

29.Sánchez-Cárdenas, MA. Anuario Observatorio Colombiano de Cuidados Paliativos. Universidad El Bosque - Observatorio Colombiano de Cuidados Paliativos. 2017.

https://doi.org/10.13140/RG.2.2.18401.92001 